\title{
Bouveret Syndrome
}

\author{
Ayca Calbay*, Ahmet Toksoy, Omer Faruk Gemis, Fatma Tortum \\ Department of Emergency, Ataturk University, Erzurum, Turkey
}

*Corresponding Author: Ayca Calbay, Department of Emergency, Ataturk University, Erzurum, Turkey, Email:drycshn@gmail.com

Abstract: Bouvet's syndrome is a common clinical condition in elderly patients. It is a gastric outlet obstruction caused by impaction of a gallstone that passes through a cholecystoduo denal orcholecystogastric fistula and because of a bowelileus. This clinical condition is associated with high morbidityandmortality.

Keywords: Bouveret syndrome; Gallstone ileus; Bilioenteric fistula

\section{INTRODUCTION}

Bouveret syndrome is a gastric outlet obstruction caused by impaction of a gallstone that passes through a cholecyst duodenal or cholecysto gastric fistula and gallstone ileus is a rare complication of cholelithiasis. Endoscopic extraction is the preferred for the theraphy. Although gallbladder lithiasis is a common disease, however, a Bouveret syndrome represents an uncomman complication.

İleus is an occlusion or paralysis of the bowel action for the passage of the intestinal contents. This situation can occour either to mechanical Or to functional ileus. Mechanical ileus is a common complication after previous surgery. The other causes of the mechanical ileus including external compression, blockage of the gastrointestinal lumen and modification in the bowel wall (tumor, inflammation/infection) [1]. Small-bowel ileus is usually due to adhesions from prior surgery $(65 \%)$ or hernia $(15 \%)$, while large-bowel ileus is usually due to cancer (70\%) or to adhesions and stenoses after recurrent diverticulitis (upto 10\%) [2].

Co-existent of Bouveret syndrome and Gallstone ileus is rare as 3\% - 6\% [3].

\section{CASE RePort}

A 59 year old man admitted to emergency service with the complaint of abdominal pain and emezis. The patient did not have a gas-gaita outlet for a week. Vital signs of the patient was as fallowing, TA: $96 / 45 \mathrm{mmHg}$, Pulse: $102 / \mathrm{min}$, Fewer: $36,5^{\circ} \mathrm{C}$ and $\mathrm{O} 2$ Sat: $85 \%$. Liver cirrhosis has been on his medical history. On physical examination, loung sounds were bilaterally decreased and ralles were heard on bases. Acid, sensitivity and defense were present in the abdominal examination. His consciousness was confused, oriented and co-operated. On his lab

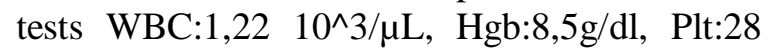

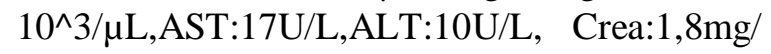
dl, Na:138mmol/l, K:4,56mmol/l, Glu:98 mg/dl, INR: 1,67. Porcelain pouch and portal vein thrombus had showned in main portal vein on abdominal ultrasonography (USG). Abdomen computarized tomography (CT) taken from the patient for the suspicion of acute abdomen clinic with ileus. So ileus observed in the patient with bilioentericfistula on CT scans [Figure 1]. After the diagnose of Bouveret Syndrome the patient hospitalized in the general surgery intensive care unit.

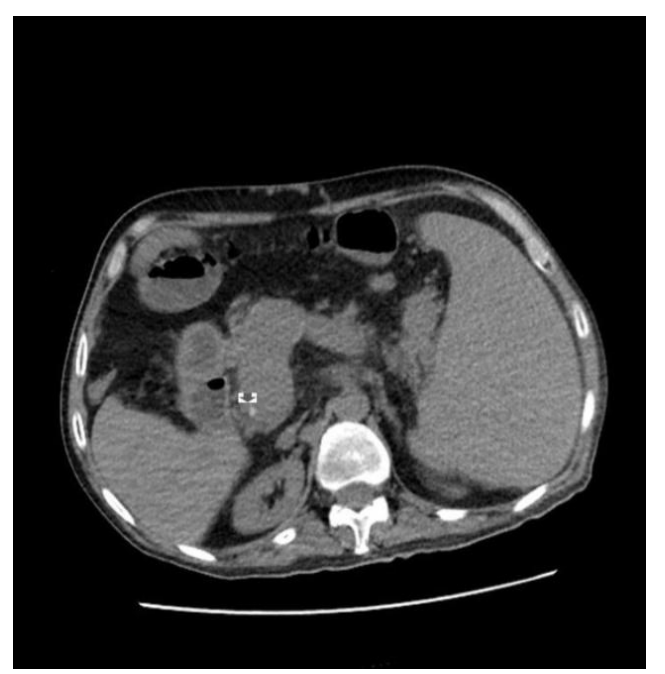

Figure1. Ileus observed in the patient with bilioenteric fistula on CT scan 


\section{DISCUSSION}

Gallstone ileus as a complication of cholecysto lithiasis is a well-described situation in old patients. It is the result of chronic inflammation of the gallbladder. The insidans of this clinic after cholecystolithiasis operations is $4.8 \%$ [4]. Bilioenteric fistulas, cholecystocolonic and cholecysto gastric fistulas can be seen after this chronic irritation. The cholecystoenteric fistulas seen in $0.3-0.5 \%$ of the patients with cholelithiasis, the majority as cholecysto duodenal (60\%) [5]. The gallstones can occur via the fistula or the opposite of this situation is also correct.

The large gallstones may block the intestinal passage by mechanical obstruction and cause gallstone ileus [6].

The main presenting symptoms for a Bouveret syndrome are nausea, vomiting and abdominal pain. The physical examination may signs for evidence of mechanical ileus. The bowel sounds are classic in the early phase and they are decreased and also absent. Murphy sign may help for the suspicion of cholelithiasis. The imaging Rigler triad (duodenal obstruction with a dilated stomach, pneumobilia and ectopic gallstone in the duodenum) is important for the diagnosis.

Endoscopy (with or without lithotripsy) is usually the first therapeutical choice, particularly in patients with have not comorbidities, but surgery has the highest achievement rate. Cholecystoenteric fistulas that have been treated by laparoscopy has been showned on literature. The physicians coincide the $4.8 \%$ of major post operation complications and $11.1 \%$ of minör postoperation complications after the laparoscopy [7].

The mortality of the patients with Bouveret syndrome without duedonal perforation is $25 \%$ of the patient and the prognosis of these patients depends on the comorbidities of the patient, the advanced age and the delay in the diagnosis[8].

\section{CONCLUSION}

In this case we want to share the possibility of migration of a large gallstone in to the duodenum with a bilioenteric fistula and cause of an ileus. The diagnosis of Bouveret syndrome depends on a clinical suspicion. İmaging methods may increase the patients early diagnose chance. İt will be reduce the mortality and complications after surgery.

\section{REFERENCES}

[1] Vilz TO, Stoffels B, Strassburg C, Schild HH, KalffJC.İleus in Adults. Dtsch ArzteblInt. 2017 Jul 24;114(29-30): 508-518. doi:10.3238/arzt ebl.2017.0508.

[2] Drozdz W, Budzynski P. Change in mechanical bowel obstruction demo graphic and etiological patterns during the past century: observations from one health care institution. Arch Surg. 2012;147:175-180.

[3] Beuran M, Venter DM, Ivanov I, Smarandache R, IftimieNastase I, Venter DP. Gallstoneileus. Annals of Academy of Romanian Scientists 2012; 3(1):5-28.

[4] Clavien PA, Richon J, Burgan S, Rohner A (1990) Gallstoneileus. Br J Surg77:737-742

[5] Lowe AS, Stephenson S, Kay CL, et al. Duo denal obstruction by gallstone (Bouveret's syndrome): A review of the literature. Endoscopy 2005; 37:82-7. DOI: 10.1055/s2004-826100

[6] Langhorst J.,Schumacher B., Deselaers T., Neuhaus H. Successful endoscopictherapy of a gastricoutlet obstruction due to a gallstone with intra corporeallaserlithotripsy: a case o Bouveret's syndrome. Gastrointest Endosc. 2000;51(2):209-213.

[7] Costi R, Randone B, Violi V, Scatton O, Sarli L, Sourbrane O, Dousset B, Montariol T (2009) Cholecysto colonicfistula: facts and myths. Are view of the 231 published cases. J Hepato biliary PancreatSurg 16:8-18

[8] Crespo-Pérez L, Angueira- Lapeña T, Defarges- Pons V, et al. Una causainfrecuente de obstruccióngástrica: síndrome de Bouve-ret. Gastroenterol Hepatol 2008; 31: 646-51. DOI: 10.1016/S0210-5705 (08) 75 813-8.

Citation: Ayca Calbay, Ahmet Toksoy, Omer Faruk Gemis, Fatma Tortum. Bouveretsyndrome. ARC Journal of Clinical Case Reports. 2017; 3(4):8-9. doi:dx.doi.org/10.20431/2455-9806.0304002.

Copyright: () 2017 Authors. This is an open-access article distributed under the terms of the Creative Commons Attribution License, which permits unrestricted use, distribution, and reproduction in any medium, provided the original author and source are credited. 